\title{
AC 2008-1657: VIDEO LECTURE ON THE PYTHAGOREAN THEORY
}

Bertram Pariser, Technical Career Institute, Inc.

Cyrus Meherji, Technical Career Institute, Inc. 


\title{
Video Lecture on PYTHAGOREAN THEORY
}

\begin{abstract}
Pythagoras derived the famous equation $a^{2}+b^{2}=c^{2}$. This discovery enabled the Greeks to build the Acropolis and the Parthenon. This equation is probably the most famous equation in mathematics. There are hundreds of proofs to the Pythagorean Theorem in mathematical literature.

My derivation of "A GEOMETRICAL PROOF OF PYTHAGORAS' THEOREM" ${ }^{1}$ is difficult for our students to understand. Students, who use the video, express a satisfaction on being able to stop, rewind and restart difficult concepts. By viewing the lecture again and again, they developed a greater understanding of the proof. The video is available 24/7 online throughout the world. All one has to do is go to www.tcicampus.net/userfolder/bpariser and click on Video Lectures.
\end{abstract}

By applying video technology, a video lecture was created and uploaded to a webpage. After hearing the lecture in class, students logon to the webpage and watch the video over and over again until they are comfortable with deriving the equation. The challenge of making a video and compressing it so that it can be seen on the Internet is considerable. While commercial studios can make movies with expensive cameras the quality of videos with a moderate camera is poor. Several attempts with different formats were explored since we have a limited amount of space on our server. The challenge was to make the video in a file format that uses the least amount of memory but does not sacrifice the quality. In a short period of time the entire proof can be understood and written using symmetry and logic. The video is well received by our students and provides a repetition of what is covered in class. At a 2 year college level we are not set up to provide recitation sessions and this is an alternate way to addressing this problem. In disseminating the material in this fashion, it not only helps our students but the rest of the world that would like to partake in it.

When teaching mathematics my students often have trouble listening to the lecture and taking notes at the same time. While I do the derivation with my students and often repeat them they have difficulty producing a proof on an examination. Since our department is accredited by TAC-ABET we are constantly looking at outcomes and assessment. Because of the challenge of increasing the output we tried extra homework, and review sessions with moderate success. One student said "Hey Dr. P why don't you make a video". I liked the idea and tried several different attempts. While the videos were good, the size of the videos on CD's was too large to be placed on the web. Since each of our students has a different internet service and different CPU in his/her computer we had to make a video which could be downloaded easily and run on computers that were not state of the art.

The video was made using a Panasonic digital video camera, it uses a DV Cassette. The original video was uploaded using Pinnacle Software and some editing was done. The last step was to save it in the format of our choosing. We selected WMV (Windows Media) originally. The file was approximately $220 \mathrm{MB}$ and later we decided that if additional videos were to be made by storage would be a problem. Because there is a storage limitation on our college server, we decided to convert the video from WMV to AVI (Audio Video Interleave) format. This conversion saved us a lot of memory. The WMV format was $220 \mathrm{MB}$ and the same video using AVI format was $108 \mathrm{MB}$. We also tried to convert it to the MP4 format which is approximately, 166MB. The MP4 uses H.264 compression and offers a better quality at a significantly low bit rate. This saved us a great deal of storage space and it is much faster to view, 
since it takes less bytes and buffers into memory faster. The video created is of good quality and clear enough for the student to view. The sound quality could be improved by using an external microphone and built in noise reduction.

Pythathe

Windows Media Audio' Wideo File

$220,024 \mathrm{~KB}$

The windows media file is around 220MB

PythaThe_NEW

Wideo Clip

$108,427 \mathrm{~KB}$

The windows AVI (Audio Video Interleave) file is around 108MB

PythaThe_NEW.m4v

M4V File

$166,636 \mathrm{~KB}$

The MP4 file format is around 166MB (uses H.264, 30fps, 640x480)

The intent of the H.264/AVC project was to create a standard capable of providing good video quality at substantially lower bit rates than previous standards (e.g. half or less the bit rate of MPEG-2,

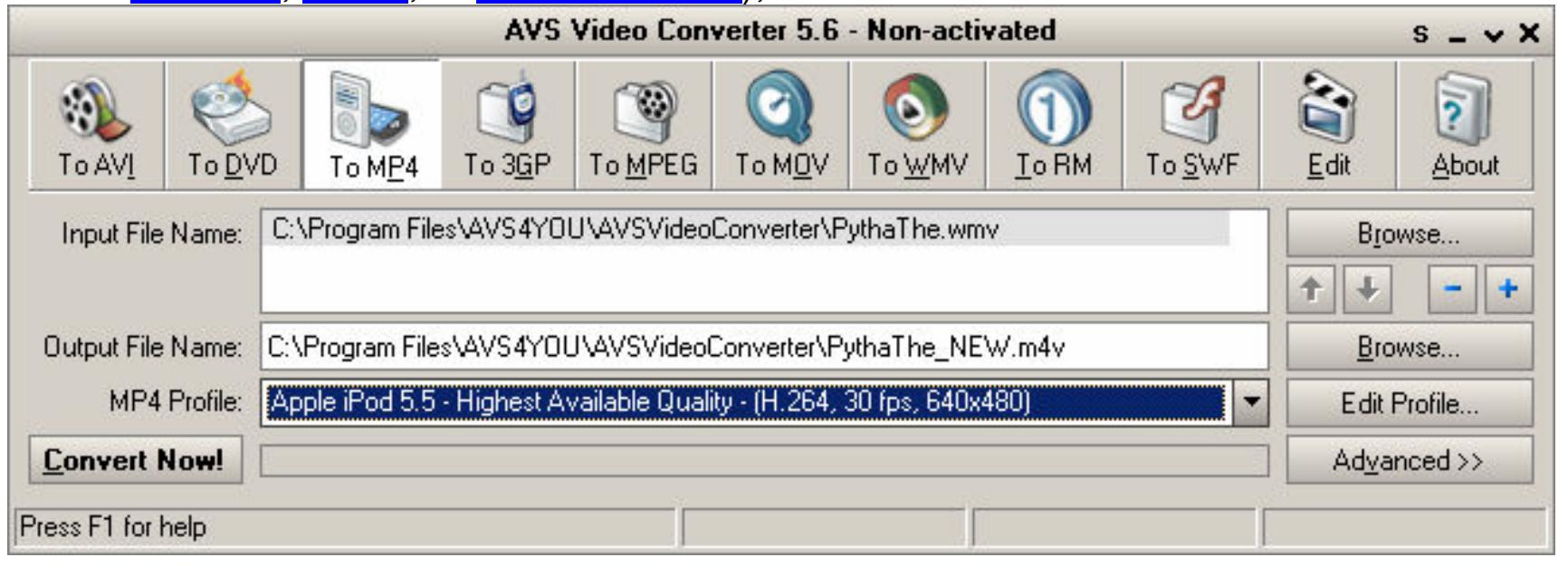

The video conversions were done using the free download from AVS Video Convertor $5.6^{3}$

The video lecture is on the web and can be seen by going to http://www.tcicampus.net/userfolder/bpariser and clicking on Video Lectures. Our students like the idea that they can slow down the lecture, stop and rewind the lecture and go over it until they get it. The lecture is well received and this TAC-ABET outcome objective has increase from 50\% to $75 \%$ 
Bibliography

1. "A GEOMETRICAL PROOF OF PYTHAGORAS' THEOREM" By Bertram Pariser, ASEE 1999. 3365

2. http://www.fileinfo.net Information on any kind of file type and their file extensions.

3. http://www.avsmedia.com/VideoTools/index.aspx AVS Inc. Video Conversion Tools.

\section{$\underline{\text { AUTHORS }}$}

Dr. Bertram Pariser is a faculty member in the Electronics Engineering Technology program at TCI, The College for Technology. He has published 23 papers and serves as the Faculty Advisor to Tau Alpha Pi National Honor Society. He Co-Founded 5 venture companies, and as a management consultant successfully catalyzed over $\$ 100$ million of new shareholder value in client businesses. He has a Ph.D. from Columbia University in Electrical Engineering \& Plasma Physics, and BS in Electrical Engineering from MIT. Bert.pariser@tcicollege.edu

Cyrus Meherji is a faculty member in the Electronics Engineering Technology and Information Technology Departments. His primary responsibilities are teaching computer programming and network infrastructure.cmeherji@tcicollege.edu 